\title{
Relato de Caso: Metástase de Carcinoma de Mama para o Intestino Grosso
}

\author{
Case Report: Metastasis from Breast Cancer to the Large Bowel \\ LÚCIO SARUBBI FILLMANN FSBCP'; CLÁUDIA MÜLLER PINHO; HENRIQUE SARUBBI FILLMANN TSBCP'; \\ ÉRICOERNESTO PRETZEL FILLMANNTSBCP ${ }^{1}$ \\ ${ }^{1}$ Proctologistas do Serviço de Colo-proctologia do Hospital São Lucas da PUCRS; ${ }^{2}$ Médica ginecologista.
}

\begin{abstract}
FILLMANN LS; PINHO CM; FILLMANN HS; FILLMANN EEP. Relato de Caso: Metástase de Carcinoma de Mama para o Intestino Grosso. Rev bras Coloproct, 2007;27(4): 456-458.

Resumo: O envolvimento metastático do trato gastrointestinal é relativamente infreqüente. Tumores malignos de mama, entretanto, podem muito raramente produzir metástases para o intestino. Relatamos aqui o caso de uma paciente com quadro clínico compatível com o de um abdômen agudo obstrutivo submetida a laparotomia exploradora com colectomia. $\mathrm{O}$ exame anátomopatológico da peça cirúrgica revelou tratar-se de um carcinoma metastático, sendo que a história clínica da paciente indicava o tratamento cirúrgico prévio de um carcinoma lobular de mama. Nossa revisão da literatura demonstrou que o carcinoma lobular, dentre as neoplasias malignas da mama, é o que mais freqüentemente leva ao comprometimento metastático do intestino, sendo o estômago o local mais afetado. Como a disseminação sistêmica é comum nestes casos, o tratamento conservador, quando possível, é o mais indicado, sendo a cirurgia reservada para as complicações.
\end{abstract}

Palavras chave: Carcinoma de mama; metástase gastrointestinal; Obstrução intestinal.

\section{INTRODUÇÃO}

O carcinoma de mama é um dos tumores malignos mais freqüentes no sexo feminino $(1,2)$. Indivíduos portadores desta doença encontram-se em situação de risco para o desenvolvimento de mestástases à distancia, mesmo após longos períodos de remissão (2). Mais freqüentemente, tais recorrências ocorrem no pulmão, ossos, sistema nervosos central e fígado (3). Entretanto, raramente, uma paciente tratada por uma neoplasia primária de mama e que se apresenta com quadro clínico sugestivo de um novo tumor do trato gastrointestinal pode, de fato, estar desenvolvendo uma recorrência metastática $(1,3,4)$. Relatamos a seguir um caso de metástase de carcinoma de mama para o cólon sigmóide.

\section{RELATO DE CASO}

Uma paciente de 91 anos foi atendida no Hospital São Lucas da PUC-RS com quadro clínico de dificuldade para eliminação de gases e fezes, com piora acentuada nas últimas 24 horas antes da consulta. Referia também desconforto abdominal com dor do tipo cólica e náuseas. Ao exame, observava-se distensão abdominal, ruídos hidroaéreos aumentados, sem sinais de irritação peritoneal. Ao toque retal, a ampola estava vazia, sem massas palpáveis.

Foi solicitado Rx de abdômen agudo que demonstrou moderada distensão do cólon, sem dilatação significativa do intestino delgado. Imediatamente, realizou-se enema contrastado que evidenciou área de estenose localizada no cólon sigmóide que ocluía a luz

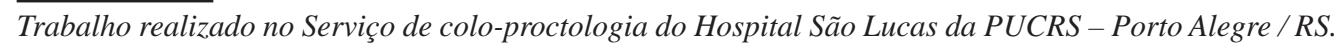

$\overline{\text { Recebido em } 27 / 06 / 2007}$

Aceito para publicação em 08/08/2007 
intestinal, imagem esta descrita como neoplasia maligna do intestino grosso obstruída.

A paciente foi submetida à intervenção cirúrgica de urgência frente a um quadro de abdômen agudo obstrutivo. Na revisão da cavidade peritoneal, observou-se lesão de aspecto tumoral, infiltrativa, ao nível do cólon sigmóide, de aspecto compatível com neoplasia primária do intestino. Não foram identificadas outras alterações sugestivas de disseminação metastática para outros órgãos intra-abdominais ou peritôneo. Realizou-se a ressecção do cólon sigmóide com exteriorização do cólon proximal através de colostomia terminal e fechamento do coto distal do intestino grosso. A paciente teve evolução pós-operatória favorável.

O exame anatomopatológico da peça cirúrgica revelou lesão nodular de $4,8 \mathrm{~cm}$ de diâmetro comprometendo a parede do intestino grosso sem ultrapassar a serosa. Sua extensão em direção à luz intestinal não ultrapassava o relevo mucoso, estando este ainda preservado. Associada a esta lesão, identificavam-se inúmeros óstios diverticulares. A análise microscópica da lesão evidenciou tecido neoplásico ocupando a camada muscular do cólon, sem ultrapassar a serosa, com extensão à submucosa, sem invadir a mucosa, sugestivo de carcinoma metastático.

A paciente relatava história de mastectomia parcial com esvaziamento axilar realizados 5 anos antes para o tratamento de um carcinoma lobular de mama. A lesão apresentava aproximadamente $2,5 \mathrm{~cm}$ $\mathrm{X} 1,5 \mathrm{~cm}$ de tamanho. Do tecido axilar, removeram-se 11 gânglios linfáticos, nenhum deles comprometido por neoplasia.

\section{DISCUSSÃO}

O câncer de mama é a neoplasia mais freqüentemente diagnosticada em mulheres no Brasil (5). A sua progressão de doença localizada para sistêmica geralmente se manifesta através de recidivas principalmente nos pulmões, ossos, cérebro e fígado (3). Alguns relatos na literatura, entretanto, chamam a atenção para o fato de que, menos freqüentemente, recidivas tumorais de carcinomas de mama podem mimetizar tumores primários do trato gastrointestinal $(1,3,4,6,7,8)$.

Schwartz e colaboradores (4) publicaram em 1998 uma série de 7 pacientes tratadas por câncer de mama, todas do sexo feminino, com média de idade de
66 anos, que se apresentaram com doença gastrointestinal sugestiva de neoplasia maligna e que na evolução do tratamento tiveram o diagnóstico de metástases do tumor primário do seio. O tempo médio entre o diagnóstico da lesão primária e o diagnóstico da metástase foi de 6 anos. Quatro casos tinham o diagnóstico histopatológico de carcinoma lobular, um caso de carcinoma ductal e 2 casos de apresentação intermediária, com componentes ductais e lobulares. Nesta série, o estômago foi o segmento do trato gastrointestinal mais afetado, com 6 dos 7 casos descritos. O cólon foi o sítio envolvido no caso restante. Endoscopicamente, a ulceração da mucosa se mostrou um evento raro, já que a recidiva tem localização preferencial na camada submucosa e muscular. $\mathrm{Na}$ análise histopatológica, observaram-se colunas de células infiltrando o plano muscular, achado este descrito como células em "fila indiana". Nesta série, $90 \%$ das pacientes apresentavam evidência de recorrência da neoplasia de mama em outros órgãos além do intestino o que se mostrou significativo no planejamento da abordagem terapêutica, optando-se por tratamentos sistêmicos e reservando a cirurgia para complicações como sangramento, obstrução ou perfuração intestinais.

Na literatura é possível identificar alguns relatos individuais de metástases de carcinomas de mama para o intestino grosso. Yokota e colaboradores (3) apresentaram, no ano 2000, o caso de uma paciente de 57 anos tratada por um carcinoma ductal invasor e que, dez anos após a cirurgia, desenvolveu uma recorrência no cólon ascendente. Rabau e colaboradores (1) relataram, em 1988, o caso de uma paciente de 53 anos tratada por um carcinoma lobular de mama que, após 7 anos do diagnóstico da lesão primária, apresentou uma recorrência metastática ao nível do íleo terminal e ceco.

Tais relatos individuais refletem o fato de que o cólon, raramente é envolvido por doença metastática de qualquer origem $(1,3,4)$. Estima-se que a incidência de metástases gastrointestinais de neoplasias mamárias chegue a no máximo $18 \%$ dos casos analisados (4). Nestes pacientes, o estômago parece ser o segmento mais freqüentemente comprometido por metástases (1). Raramente, o tubo digestivo é o único sistema envolvido pela disseminação tumoral, sendo comum o diagnóstico simultâneo de lesões em outros órgãos e sistemas (4). Apesar de o tipo histológico mais comum do câncer de mama ser o carcinoma ductal invasor, o tipo lobular é o 
Rev bras Coloproct

Outubro/Dezembro, 2007
Relato de Caso: Metástase de Carcinoma de Mama para o Intestino Grosso

Lúcio Sarubbi Fillmann e Cols.
Vol. 27 que apresenta maior tendência a disseminação para o intestino (4,9). Tais tumores mais freqüentemente envolvem o peritôneo e retroperitôneo, e , na análise microscópica, observa-se, seguidamente, células em anel de sinete (3). O diagnóstico pode ser difícil, uma vez que a mucosa intestinal freqüentemente está preservada, com o desenvolvimento da recorrência acontecendo no pla- no muscular e submucoso, fato este que compromete a eficiência da biópsia endoscópica (3). Uma vez que, na maioria das pacientes, múltiplas metástases em diferentes órgãos são diagnosticadas, deve-se considerar que um tratamento sistêmico é o mais indicado, reservandose a cirurgia para situações especiais, especialmente nas complicações intestinais $(1,4)$.

\begin{abstract}
Gastrointestinal manifestations of breast cancer are not commom. We report a case of a female patient who was submitted to a laparotomy with clinical and radiological signs of large bowell obstruction. Her clinical hitory showed that she was treated for breast cancer several years before. Pathology indicated that a metastatic carcinoma within the bowell wall was the cause of the obstruction. Previous reports described the lobular carcinoma of the breast as the most commom type of breast cancer to produce bowell metastases. The stomach is described as the most frequent site of this metastatic dissemination. Since sistemic disease is common within these patients, surgical ressection is indicated only in the presence of complications like obstruction or bleeding.
\end{abstract}

Key words: Breast carcinoma; metastatic carcinoma; intestinal obstruction.

\section{REFERÊNCIAS}

1. Rabau MY, Alon RJ, Werbin N, et al. Colonic metastases from lobular carcinoma of the breast: report of a case. Dis Colon Rectum 1988; 31: 401-2.

2. Edwards BK, Brown ML, Wingo PA, et al. Annual report to the nation on the status of cancer, 1975-2002, featuring population-based trends in cancer treatment. J Natl Cancer Inst 2005;97:1407-27.

3. Yokota T, Kunii Y, Kagami M, et al. Metastatic breast carcinoma masquerading as primary colon cancer. Am J Gastroenterol 2000; 95: 3014-5.

4. Schwartz RE, Klimstra DS, Turnbull AD. Metastatic breast cancer masquerading as gastrointestinal primary. Am J Gastroenterol 1988; 93: 111-4.

5. Instituto Nacional do Câncer-Ministério da Saúde do Brasil. Estimativas da incidência e mortalidade por câncer no Brasil 2006. http:// www.inca.org.br, março de 2006.

6. Voravud N, El-Naggar AK, Balch CM et al. Metastatic lobular breast carcinoma simulating primary colon cancer. Am J Clin Oncol 1992; 15: 365-9.
7. Taal BG, Jager H, Steinmetz R et al. The spectrum of gastrointestinal metastases of breast carcinoma: I. Stomach. Gastrointest Endosc 1992; 38: 130-5.

8. Taal BG, Jager H, Steinmetz R et al. The spectrum of gastrointestinal metastases of breast carcinoma: II. The colon and rectum. Gastrointest Endosc 1992; 38: 136-41.

9. Gegundez C, Paz JM, Mata I, Cao J, Conde J, Couselo JM, Avila S, Arija F. Small bowell obstruction due to metastatic lobular carcinoma of the breast. Cir Esp 2005; 77(2):102-4.

10. Aurello P, D'Angelo F, Cosenza G, Petrocca S, Stopacciaro A, Ramacciato G, Ziparo V. Gastric metastasis 14 years after mastectomy for breast lobular carcinoma: case report and literature review. Am Surg 2006; 72(5): 456-60.

\section{Endereço para correspondência:}

LÚCIO FILLMANN

Avenida Ipiranga 6690 sala 307; Centro Clínico PUCRS

Porto Alegre/RS

90610-000 